\title{
Research on Overhead Line Cost Prediction Based on Index Construction
}

\author{
Yongping $\mathrm{He}^{1}$, Xiaomin Liu ${ }^{1}$, Kai Yang ${ }^{1}$, Shangke Liu ${ }^{1}$ and Yaxuan Chen ${ }^{2 *}$ \\ ${ }^{1}$ Institute of Economy and Technology, Ningxia Electric Power Co., Yinchuan, Ningxia, 750000, China \\ ${ }^{2}$ School of Economics and Management, North China Electric Power University, Beijing, 102206, China
}

\begin{abstract}
In order to meet the growing demand of the society for electric power, the construction of electric power infrastructure is constantly carried out. However, investment in construction and cost control are particularly important. Because of its own characteristics, overhead line project cost control management is difficult to carry out. According to the characteristics of overhead line engineering, this paper constructs an overhead line engineering cost index system composed of 24 indexes including voltage grade from three aspects: technical conditions, engineering quantity attribute and cost attribute. Combining with the actual data of the completed project, the BP neural network algorithm is used to predict the static investment of the project. The accuracy of the prediction model reaches $99.9 \%$. This verifies the rationality and comprehensiveness of the overhead line project cost index system constructed in this paper, and provides reliable guidance for the overhead line project cost management.
\end{abstract}

\section{Introduction}

As one of the important contents of power infrastructure construction, the development of power transmission and transformation projects is of great significance to ensure the smooth operation of economy. At the same time, some problems in the construction of power transmission and transformation projects are gradually exposed, such as large investment, low efficiency and loopholes in cost management. From the point of view of Engineering characteristics, it is difficult to determine and control the cost of overhead line project in power transmission and transformation projects because of its wide spanning area, complex construction environment and many unpredictable factors. In order to effectively improve the level of cost control, the first task is to quickly and accurately determine the project cost, and the importance of project cost prediction is self-evident.

At present, some scholars have carried out research on investment cost prediction, mainly using grey correlation analysis, support vector machine and BP neural network algorithm. Kim S uses the hybrid analytic hierarchy process (AHP) and case-based reasoning (CBR) to study the cost estimation model in the early stage of highway project development in Korea [1]. Wang Fuping combines the intelligent learning algorithm with the actual situation of power transmission and transformation projects, considers the influence of control line on design optimization, and puts forward the refinement method of control line for power transmission and transformation project cost. Based on Chebyshev inequality, Zhang Geli et al. constructed a transmission and transformation project cost prediction model, which expanded the dimension of cost analysis. Ling Yunpeng et al. proposed a method of transmission line project cost prediction based on BP neural network, and established a BP neural network prediction model with three-layer structure [4]. Wang Xin et al. based on the actual cost data of overhead line of power grid, proved that the improved PSO-SVM model can effectively predict the cost of overhead line of power grid, and the average error of the prediction results is small [5].

The existing research results provide sufficient theoretical support for this paper, but with the continuous improvement of power engineering cost system and the rapid development of new technologies such as big data, the control of overhead line project cost is lack of systematicness, and there is no systematic and directly applicable engineering cost index system. Therefore, the study of overhead line project cost prediction based on index construction has certain theoretical value and practical guiding significance.

\section{Theoretical basis}

\subsection{Data Mining}

Data mining usually extracts implicit, unknown, potentially interesting and potentially valuable knowledge and rules from a large amount of data through various algorithms. It is mainly based on artificial intelligence,

\footnotetext{
${ }^{*}$ Corresponding author's e-mail: 15010979128@163.com
} 
machine learning, pattern recognition, statistics, database and so on. It highly automates the analysis of large amounts of data, makes inductive reasoning, and then excavates the potential.

In this paper, data mining principle is used to process a large number of historical data of overhead substation projects, and then to mine the hidden information in the data, especially to provide support for the construction of cost indicators of substation projects.

\subsection{BP Neural Network}

BP neural network is a multi-layer feedforward network trained by error back propagation. Its algorithm is called BP algorithm. Its basic idea is gradient descent method. Gradient search technology is used to minimize the mean square error of the actual output value and the expected output value of the network. It is not necessary to determine the mathematical equation of the mapping relationship between input and output in advance. Only through self-training, we can learn some rules and get the results closest to the expected output value when given the input value.

In this paper, the BP neural network algorithm is used to make an empirical analysis based on the actual project data. The static investment of the project is forecasted on the basis of calculating the project cost index, so as to verify the rationality of the index system construction.

\section{Construction of Index System}

\subsection{Ideas on Construction of Overhead Line Engineering Indicators}

In the context of the wide application of new technologies such as big data and cloud computing, it is particularly important to collect and excavate engineering data information in line with the inevitable requirement of the trend of "Internet + " development. Collecting cost data based on completed projects can not only realize the value of reuse and mining of completed project data, but also lay a good foundation for the prediction and control of project cost.

Based on the characteristics of China's overhead line projects and the analysis of historical engineering cost data of completed overhead line projects, this paper constructs an index system of engineering cost from three aspects: technical condition attributes, engineering quantity attributes and cost attributes.

\subsection{Establishment of Cost Index System for Overhead Lines}

The factors affecting the cost of each unit project generally involve the attributes of technical conditions. In this paper, eight attributes such as voltage level, topographic comprehensive coefficient, geological comprehensive coefficient, conductor type, conductor cross-section, icing and wind speed, splitting number and tension ratio are selected to establish indicators. According to the division of overhead line engineering, it is divided into six units: basic engineering, tower engineering, grounding engineering, overhead line engineering, accessory engineering and auxiliary engineering. Each unit project includes engineering quantity index and cost index. Finally, the project cost index system composed of 24 indexes is obtained, as shown in Table 1.

Table 1. Cost Index System of Overhead Line Project.

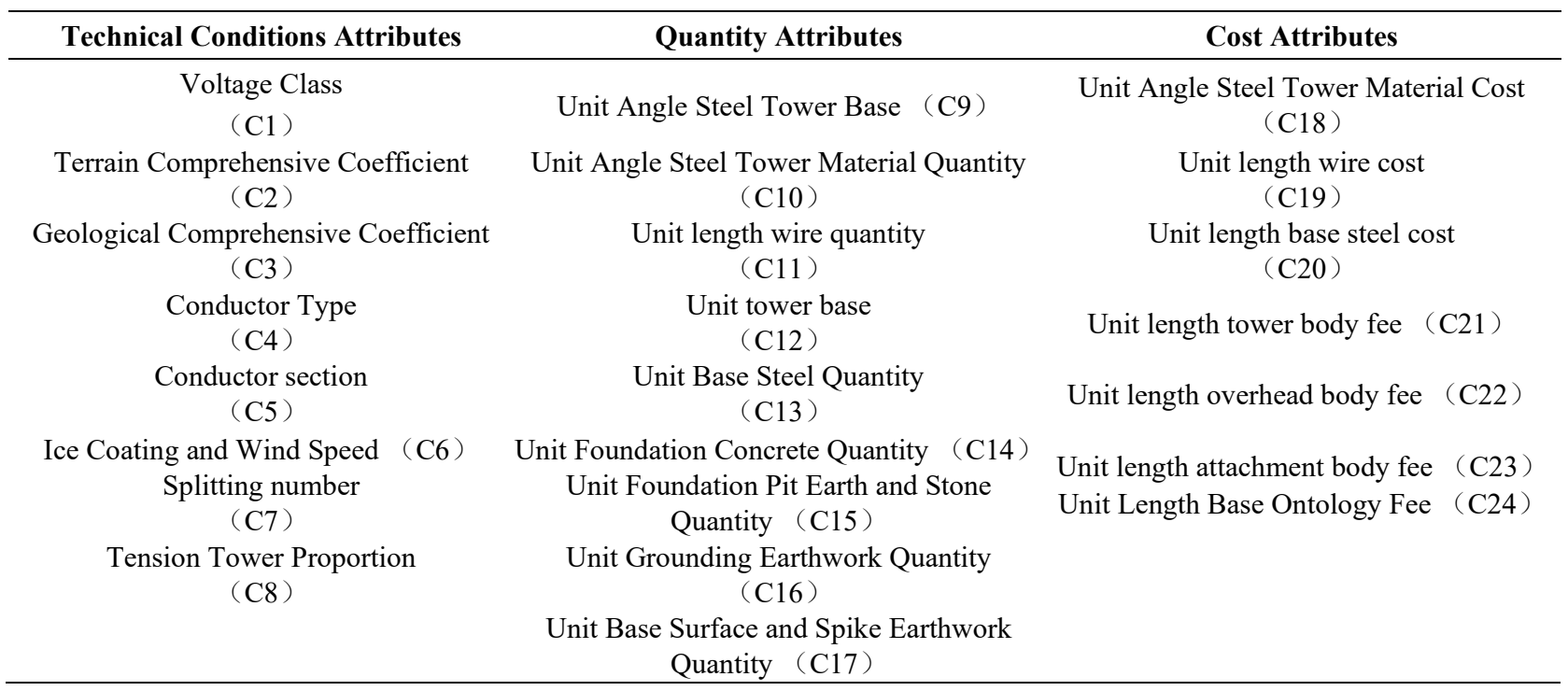




\subsubsection{Index quantification}

(1) Voltage Class

Overhead line voltage levels are commonly $35 \mathrm{kV}$, $110 \mathrm{kV}, 220 \mathrm{kV}, 500 \mathrm{kV}$. In order to facilitate statistical analysis of indicators, the voltage levels are quantified sequentially with the number of 1-4.

(2) Conductor Type

Quantification of "conductor type" is similar to "voltage grade". Common conductors are steel-core highconductivity aluminium strand, steel-core aluminium strand, aluminium alloy core conductor, heat-resistant conductor, medium-strength aluminium alloy strand and others, which are quantified by 1-6 digits in turn.

(3) Conductor section

Similar to the "voltage level" quantization, $150 \mathrm{~mm}$, $185 \mathrm{~mm}, 240 \mathrm{~mm}, 300 \mathrm{~mm}, 400 \mathrm{~mm}, 500 \mathrm{~mm}, 630 \mathrm{~mm}$ and $800 \mathrm{~mm}$ are commonly used, which are quantified by 1-8 digits in turn.

(4) Ice Coating and Wind Speed

Similar to "voltage level" quantification, ice $(<29,31$, $33,35,>37)$, light ice $(23.5,25,27,29,31,>33)$ and medium ice $(23.5,25,27,29,31,>33)$ are commonly quantified with 1-17 digits in turn.

\subsubsection{Comprehensive Coefficient Processing}

(1) Terrain Comprehensive Coefficient

According to the "Budget Quota for Power Grid Engineering" for different topographic coefficients of each sub-project, the calculation is based on formula (1).

Terrain Comprehensive Coefficient $=$ flats $\times w_{1}+$ hills $\times w_{2}+$ hydrographic net $\times w_{3}+$ swamps $\times w_{4}+$ hilly areas $\times w_{5}+$ mountains $\times w_{6}+$ deserts $\times w_{7}+$ steep mountains $\times w_{8}$

Among them, $w_{1}, w_{2}, w_{3}, w_{4}, w_{5}, w_{6}, w_{7}, w_{8}$ is the proportion of flats, hills, hydrographic net, swamps, hilly areas, mountains, deserts and steep mountains, so as to calculate the comprehensive topographic coefficients.

(2) Geological Comprehensive Coefficient

Geological conditions generally include frozen soil, common soil, hard soil, loose sand stone, water pit, mud pit, quicksand pit, rock blasting and rock manpower. Different geological conditions lead to different construction difficulties. Similar to the method of calculating comprehensive topographic coefficients, different weight coefficients are given to different geological conditions according to the experience of experts. Then, it is synthesized into a parameter according to the proportion of each geological condition.

\subsubsection{Unitization treatment}

In overhead line engineering, the length of line and the number of loops have a significant impact on the project cost. The corresponding attribute indexes of different line length and number of loops are not comparable. Therefore, based on the length of line fold as a benchmark, the formula for calculating the length of line fold as shown in formula (2), the attribute indexes are carried out. Unitization treatment.

Line folding length=length of single circuit $* 1+$ length of double circuit*2+ length of triple circuit*3+ length of quadruple circuit*4+ length of thread drawing

\section{Empirical Analysis of Cost Forecasting}

\subsection{Data Source and Processing}

This paper collects 132 overhead line projects of power grid company. According to the index system constructed above, each project has 24 indexes. The sample data of the index is shown in Table 2.

Table 2. Data Sample of Overhead Line Engineering Indicators.

\begin{tabular}{c|c|c|c|c|c|c|c|c|c|c|c|c}
\hline Sample & $\mathrm{C} 1$ & $\mathrm{C} 2$ & $\mathrm{C} 3$ & $\mathrm{C} 4$ & $\cdots$ & $\mathrm{C} 9$ & $\mathrm{C} 10$ & $\cdots$ & $\mathrm{C} 21$ & $\mathrm{C} 22$ & $\mathrm{C} 23$ & $\mathrm{C} 24$ \\
\hline 1 & 3 & 1.00 & 10.00 & 2 & $\cdots$ & 1.42 & 29.49 & $\cdots$ & 39.20 & 13.94 & 8.71 & 25.26 \\
\hline 2 & 2 & 6.77 & 25.00 & 2 & $\cdots$ & 2.98 & 17.43 & $\cdots$ & 21.76 & 7.74 & 4.84 & 14.02 \\
\hline 3 & 2 & 8.90 & 8.50 & 2 & $\cdots$ & 0.62 & 4.74 & $\cdots$ & 16.26 & 5.78 & 3.61 & 10.48 \\
\hline 4 & 2 & 1.00 & 10.00 & 2 & $\cdots$ & 5.83 & 48.03 & $\cdots$ & 5.83 & 48.03 & 3.33 & 5.83 \\
\hline 5 & 1 & 1.52 & 9.60 & 2 & $\cdots$ & 3.15 & 20.05 & $\cdots$ & 0.63 & 1.76 & 2.27 & 5.76 \\
\hline$\cdots$ & $\cdots$ & $\cdots$ & $\cdots$ & $\cdots$ & $\cdots$ & $\cdots$ & $\cdots$ & $\cdots$ & $\cdots$ & $\cdots$ & $\cdots$ & $\cdots$ \\
\hline 128 & 3 & 8.22 & 14.01 & 2 & $\cdots$ & 7.21 & 68.92 & $\cdots$ & 257.84 & 91.68 & 57.30 & 166.16 \\
\hline 129 & 2 & 1.94 & 18.26 & 2 & $\cdots$ & 2.08 & 15.84 & $\cdots$ & 23.62 & 8.40 & 5.25 & 15.22 \\
\hline 130 & 3 & 5.75 & 17.06 & 2 & $\cdots$ & 1.66 & 39.45 & $\cdots$ & 52.58 & 18.69 & 11.68 & 33.88 \\
\hline 131 & 1 & 1.08 & 20.00 & 2 & $\cdots$ & 2.76 & 7.28 & $\cdots$ & 8.87 & 3.15 & 1.97 & 5.72 \\
\hline 132 & 4 & 2.65 & 31.72 & 2 & $\cdots$ & 2.20 & 50.07 & $\cdots$ & 164.99 & 64.96 & 0.00 & 89.08 \\
\hline
\end{tabular}




\subsection{Cost Prediction Based on BP Neural Network}

In this paper, 24 indicators mentioned above are taken as input, static investment of overhead line project is taken as output, $80 \%$ of the project is set as training set, and the remaining 20\% is set as test set. BP neural network is used to predict, and a three-layer BP neural network prediction model is established as shown in Figure 1.

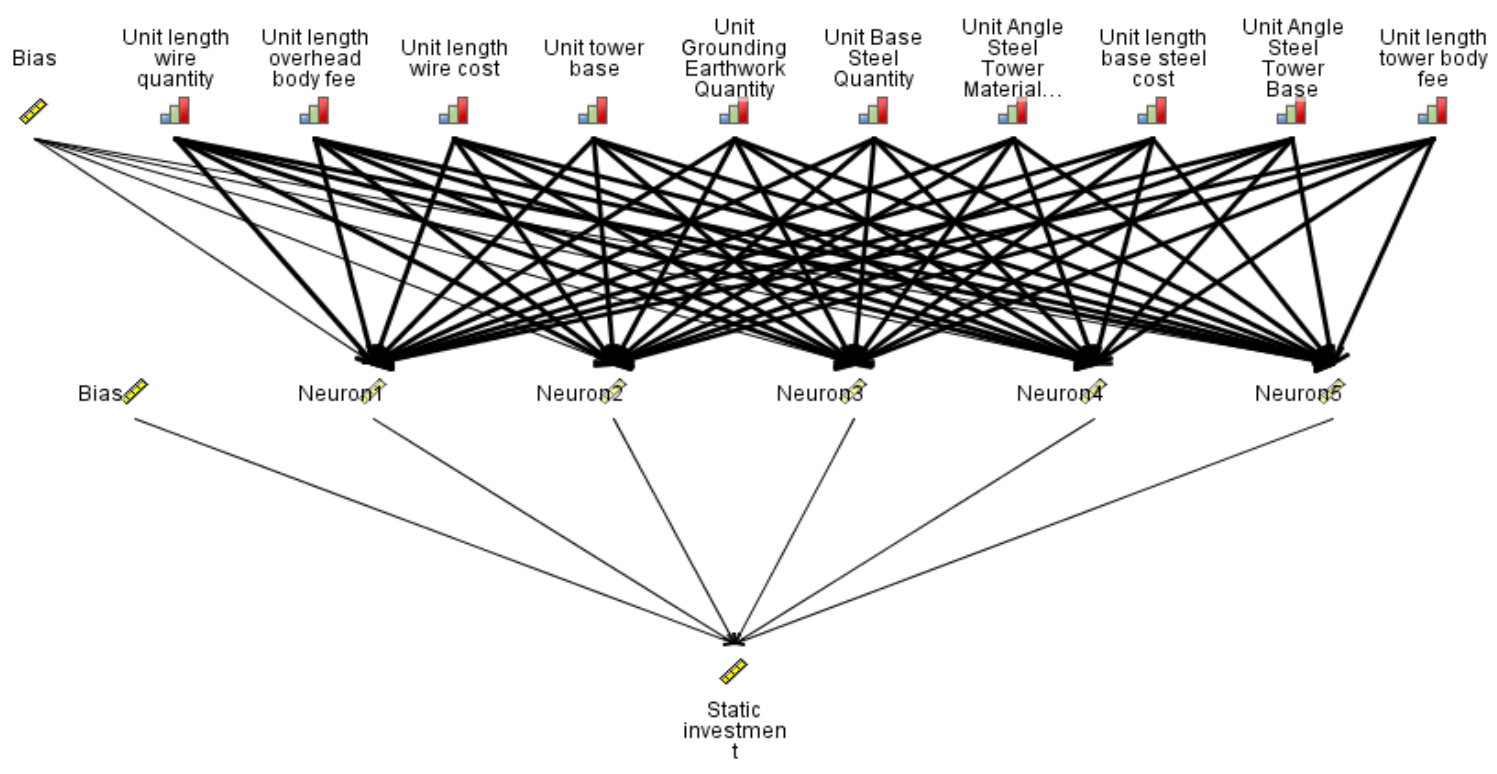

Figure 1. Schema of Neural Network.

Among the 24 variables in the input layer, the most important one is the quantity of basic steel per unit. The scatter plot composed of actual static investment and predicted value of overhead line project is shown in Figure 2.

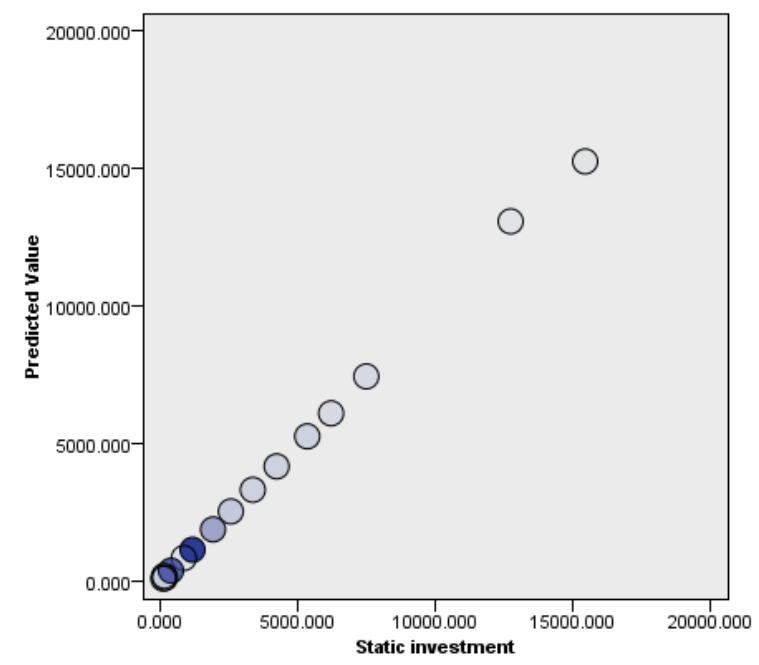

Figure 2. Scatter plot.

From the above figure, it can be seen that the points on the scatter plot composed of the actual static investment of overhead line project as abscissa and the predicted value as longitudinal coordinate are closely and uniformly distributed near the 45 degree bisector, which shows that the deviation between the predicted value and the actual value is small and the predicted effect is good. After calculation, the accuracy of the BP neural network applied to the investment prediction of overhead line project is
$99.9 \%$, which is higher, thus proving the accuracy and comprehensiveness of the index system of overhead line project cost.

\section{Conclusions}

On the basis of data mining theory and combined with the characteristics of overhead line engineering, this paper constructs an overhead line engineering cost index system, and uses BP neural network algorithm to carry out empirical analysis with actual engineering data. The main research results are as follows.

(1) The construction cost index system of overhead line is constructed. Firstly, based on the cost-related data of completed projects, the data base is formed; then, the characteristics of overhead line projects are analyzed, the overhead line projects are decomposed into unit projects, and the projects are divided; finally, the technical conditions, engineering quantity and cost indicators are summarized, and 24 indicators are obtained.

(2) An overhead line engineering prediction model is constructed and an empirical analysis is carried out. The actual data of 132 completed overhead line projects are collected and pretreated, and 24 indicators of each sample are obtained; the indicators are input of BP neural network, the static investment of the project is output of the model, and the comparative analysis between the predicted value and the actual value of the project cost is carried out. The accuracy of the results is as high as $99.9 \%$, which verifies the rationality and comprehensiveness of the overhead line project cost index system, providing effective support for improving the level of project cost control and ensuring the economic rationality of overhead line project. 


\section{References}

1. Kim S . Hybrid forecasting system based on casebased reasoning and analytic hierarchy process for cost estimation[J]. Statyba.

2. Wang FP. Subdividing Method and Applied Research of Power Transmission and Transformation Project Cost Control [D].Chongqing: Chongqing University, 2015.

3. Zhang GL, Wen WN, Li PD, et al. Reasonable Interval Calculation Method for Power Transmission Project Cost Based on Chebyshev's Inequality [J]. Electric Power Construction, 2014, 35(9):118-122.

4. Ling YP, Yan PF, Han CZ, et al. BP Neural Network Based Cost Prediction Model for Transmission Projects [J]. Electric Power,2012,45(10):95-99.

5. Wang X, Geng P, Yuan J. Study on the Prediction Model of Overhead Line Cost of Power Grid [J]. China Electric Power Enterprise Management, 2018 (24): 76-77. 